\title{
Validez y fiabilidad de la versión argentina del PCAT-AE para evaluar la atención primaria de salud
}

\author{
Silvina Berra, ${ }^{1}$ Lisiane Hauser, ${ }^{2}$ Yanina Audisio, ${ }^{1}$ José Mántaras, ${ }^{3}$ \\ Valeria Nicora, ${ }^{3}$ Mônica Maria Celestina de Oliveira, ${ }^{4}$ \\ Barbara Starfield ${ }^{5}$ y Erno Harzheim ${ }^{6}$
}

Forma de citar

Berra S, Hauser L, Audisio Y, Mántaras J, Nicora V, de Oliveira MMC, et al. Validez y fiabilidad de la versión argentina del PCAT-AE para evaluar la atención primaria de salud. 2013;33(1):30-9.

RESUMEN Objetivo. Determinar la validez y la fiabilidad de los "instrumentos para evaluar la atención primaria de salud" (Primary Care Assessment Tool [PCAT, por su siglas en inglés]), versión dirigida a usuarios adultos, adaptada para Argentina (AR-PCAT-AE).

Métodos. Se realizaron dos estudios piloto transversales con personas mayores de 17 años, mediante cuestionarios mayoritariamente autoadministrados. Se analizaron los items equivalentes a la versión original de los PCAT y otros 13 propuestos para ser adicionados. Se examinó la validez factorial y la fiabilidad de las puntuaciones de cada dimensión. Los indicadores de validez fueron: factores que tuvieran tres o más items con una carga factorial $>0,35$, correlación ítem-total >0,30 e interpretación de la solución en consecuencia con el modelo teórico.

Resultados. El análisis factorial resultó en nueve factores que explicaron 57,4\% de la variabilidad. Considerando los criterios de validez preestablecidos, se excluyeron dos items de las dimensiones de continuidad e integralidad y se adicionaron seis en integralidad y competencia cultural. En las dimensiones, el porcentaje de preguntas con correlación itemtotal mayor de 0,30 osciló entre 67\% y 100\% y el coeficiente de consistencia interna alfa de Cronbach entre 0,44 y 0,90 .

Conclusiones. El cuestionario AR-PCAT-AE tiene validez y fiabilidad adecuadas, manteniendo una alta equivalencia con la versión original. El proceso presentado podría ser adoptado en otros contextos para avanzar en la necesidad de evaluar las funciones de la atención primaria con herramientas de calidad.

Palabras clave Atención primaria de salud; encuestas de salud; servicios de salud; atención ambulatoria; Argentina.

Instituto de Investigaciones en Ciencias de la Salud (INICSA), Córdoba, Argentina. La correspondencia se debe dirigir a Silvina Berra. Correo electrónico: silvinaberra@gmail.com

2 Programa de Pós Graduação em Epidemiologia, Universidade Federal do Rio Grande do Sul (UFRGS), Brasil.

3 Escuela de Trabajo Social, Facultad de Derecho y Ciencias Sociales, Universidad Nacional de Córdoba (UNC), Córdoba, Argentina.

4 Departamento de Educação e Informação em Saúde, Universidade Federal de Ciências da Saúde de Porto Alegre, Brasil.
A nivel internacional, y particularmente en las Américas, se ha reconocido la necesidad de fortalecer la atención primaria de salud (APS) y de evaluar el cumplimiento de sus funciones (1-5).

\footnotetext{
Department of Health Policy and Management John Hopkins University, Baltimore, Estados Unidos de América.

6 Departamento de Medicina Social, Faculdade de Medicina, Universidade Federal do Rio Grande do Sul (UFRGS), Brasil.
}

Además se ha señalado que, en ambos casos, hace falta contar con herramientas idóneas (3) y se han discutido marcos conceptuales para llevar a cabo esta tarea en la Región (6). Sin embargo, los esfuerzos por evaluar el grado de implementación de las reformas han sido más bien aislados e igualmente heterogéneos (7).

La calidad del primer nivel de atención se definió como la prestación de ser- 
vicios accesibles e integrales para la mayoría de las necesidades en salud de la población, y el desarrollo de una relación con los pacientes sostenida en el tiempo, en el contexto de la familia y la comunidad (8). Starfield realizó un avance significativo en el campo de la evaluación de la APS al definir sus funciones esenciales y secundarias, distinguir entre enfoques de evaluación dirigidos a políticas o al primer nivel de atención y desarrollar un conjunto de "instrumentos para evaluar la APS" (PCAT), ${ }^{7}$ que tienen la ventaja -sobre otras herramientas similaresde que pueden obtener similar información desde distintas perspectivas (9-12). Los cuestionarios PCAT se componen de preguntas para medir las funciones esenciales de primer contacto, continuidad, coordinación e integralidad, así como las funciones secundarias de enfoque familiar, orientación comunitaria y competencia cultural (cuadro 1) (13). El conjunto de cuestionarios PCAT puede ser respondido por usuarios de servicios de APS, profesionales (proveedores), administradores de centros de salud o expertos en sistemas sanitarios.

Las versiones originales de estas herramientas —desarrolladas en Estados Unidos de América- ya han sido adaptadas y son utilizadas en Canadá (14, 15), Brasil (16-18), España (19-21), Hong Kong $^{8}$ (22), Nueva Zelandia (23) y Taiwán $(24,25)$; al momento, están siendo adaptadas en China, Filipinas, Sudáfrica y Uruguay (26). En general, los PCAT que fueron evaluados demostraron una adecuada consistencia interna y confiabilidad test-retest, y confirmaron su validez factorial acorde al modelo teórico multidimensional, con contadas excepciones en algunos ítems (10, 11, 17, 27, 28). La diseminación de este modelo de evaluación a nivel internacional permitirá a corto plazo comparar resultados entre países con características diversas. Sin embargo, para conseguirlo es importante que las versiones que se adaptan en cada país mantengan la calidad de las originales en cuanto a su validez y fiabilidad, a la vez que logren adecuarse al contexto cultural donde serán utilizadas.

A partir de mediados del siglo XX, el sistema de salud argentino se conformó en tres subsectores: i) el estatal, de cobertura universal; ii) el de obras socia-

\footnotetext{
Siglas de la denominación en inglés (Primary Care Assessment Tools).

8 Región Administrativa Especial de China.
}

les, basado en el modelo bismarkiano de seguridad social, y iii) el privado. Las reformas de las últimas décadas, a tono con las transformaciones económicas globales, consolidaron la tendencia a la fragmentación institucional y a la segmentación social (29). Actualmente, $47 \%$ de la población argentina declara cobertura en el subsector de obras sociales por afiliación obligatoria, alcanzando al $56,8 \%$ por prepaga voluntaria; un $15,5 \%$ declara cobertura prepaga en el sector privado o mixto con obra social, y un $36 \%$ estaría cubierto solo por el sector público (30).

En Argentina, las versiones del PCAT fueron adaptadas con el propósito de que puedan responder usuarios, profesionales y administradores de centros de salud, y permitir comparaciones con otros países (31). Durante el proceso de adaptación, el grupo de trabajo local consideró de interés añadir algunos ítems sobre los servicios disponibles y la orientación comunitaria de la APS, intentando captar actividades habitualmente incluidas en los lineamientos de trabajo de los equipos de salud en sus comunidades. El objetivo del presente estudio fue determinar la validez y la fiabilidad de la versión argentina del PCAT para usuarios adultos (AR-PCAT-AE)

\section{MATERIALES Y MÉTODOS}

\section{Diseño y muestras}

Se realizaron dos estudios piloto de diseño transversal que incluyeron a personas de 18 o más años de edad. El primero, en 2010, se llevó a cabo en tres institutos provinciales de educación media de la ciudad de Córdoba, seleccionados por reunir a adolescentes de nivel socioeconómico medio y bajo de diversos barrios de la ciudad, y también por la conveniencia debida a la accesibilidad y la confianza de los directivos que aceptaron participar en dicho estudio. En estos institutos no se realizó selección de una muestra, sino que se invitó a participar a 542 madres y padres de escolares de 11 a 15 años de edad que cursaban el ciclo básico unificado (CBU). Los adultos recibieron la carta con información del estudio, los cuestionarios que indagaban acerca de su atención de salud y sobres; en total fueron 275 - entre madres y padres- quienes aceptaron participar, firmaron el consentimiento informado y lo devolvieron a la escuela a través de sus hijos, junto con el cuestionario respondido en el sobre cerrado.

En el segundo estudio piloto participaron personas adultas beneficiarias de la Obra Social de la Universidad Nacional de Córdoba, la cual a julio de 2010 contaba con 56068 afiliados, mayoritariamente personal docente, no docente y jubilados o pensionados de la Universidad, junto a un porcentaje menor de adherentes. Se realizó un muestreo aleatorio simple de 500 personas, de las cuales 309 pudieron ser localizadas y, de ellas, 261 aceptaron participar. En este caso, para la administración del cuestionario se diseñaron versiones en formato de autoadministrado, en papel o digital, y versiones para ser respondidas mediante entrevistas personales o telefónicas.

El tamaño de la muestra fue de $97 \mathrm{su-}$ jetos por grupo, número necesario para comparar medias y detectar diferencias iguales o superiores a una unidad en la valoración de la APS por dimensiones, con un riesgo alfa de 0,95 , una precisión de $+/-0,3$ unidades y una desviación estándar (DE) estimada de 1,8.

\section{El instrumento}

El cuestionario implementado contenía 111 ítems para medir las dimensiones de la APS y otras para caracterizar a la fuente de APS y a la población (p. ej. estado de salud y cobertura médica). Los ítems sometidos a evaluación de la fiabilidad y la validez del cuestionario fueron 104, correspondiendo 91 a la versión original del instrumento y 13 a ítems propuestos durante la adaptación en Argentina (31). Las preguntas recogieron datos sobre la presencia de rasgos específicos de los conceptos subyacentes a cada una de las cuatro dimensiones esenciales, con sus dos subdimensiones, y las tres dimensiones secundarias del modelo teórico (cuadro 1), según la experiencia de la población. Los ítems de todas las dimensiones se responden mediante las siguientes opciones: $1=$ definitivamente no; 2 = probablemente no; 3 = probablemente sí; 4 = definitivamente sí; y 9 = no sabe/no recuerda, a la que posteriormente se le otorga el valor 2,5 (13). Los puntajes de las respuestas son usados para calcular un promedio de los valores obtenidos por los ítems de cada dimensión o atributo, obteniéndose como resultado una escala con rango de 1 a 4, que se interpreta como la valoración por parte del usuario de su expe- 
CUADRO 1. Funciones esenciales y secundarias de las dimensiones de la atención primaria de salud (APS) valoradas por los cuestionarios PCAT, ${ }^{a}$ y número de ítems en cada una correspondiente a la versión para usuarios adultos (PCAT-AE)

Esenciales

Primer contacto. Supone la idea de que cada vez que surge un problema nuevo, un centro o profesional de salud concreto debe ser la puerta de entrada al sistema y accesible a la población. Se compone de dos subdimensiones:

B. Utilización: el centro o profesional proveedor de APS es realmente visitado ante un problema o evento nuevo.

C. Acceso: es el elemento estructural necesario para el primer contacto.

Continuidad. Relación personal a largo plazo entre el usuario y el profesional o centro de salud, basada en la confianza y el conocimiento de la persona y su familia. Se compone de dos subdimensiones:

A. Grado de afiliación: medida en que los usuarios identifican una fuente habitual de atención de salud y los centros de atención son capaces de identificar a la población elegible o candidata a sus servicios.

D. Continuidad interpersonal: atención longitudinal y focalizada en la persona (no en la enfermedad).

Coordinación. Es la función de enlace entre los servicios de atención, de tal modo que la persona reciba un cuidado apropiado de todos sus problemas de salud bajo la responsabilidad de su proveedor de APS. Esta dimensión permite conocer problemas anteriores y nuevos, e incluye también la referencia y el acompañamiento de la atención realizada en otros servicios especializados. Se compone de dos subdimensiones:

E. Integración de la información: mecanismos de transferencia de la información y de recepción de otras fuentes que pueden estar involucradas en la atención de las personas.

F. Sistemas de información: disponibilidad de instrumentos de registro de la información y su incorporación al plan de atención de la persona, así como el desarrollo e implementación de un plan apropiado de identificación y gestión de necesidades de la población.

Integralidad o globalidad. Posibilidad de ofrecer un catálogo extenso, articulado e integral de todos los servicios que la población necesita. Incluye la identificación de problemas de todo tipo, sean orgánicos, funcionales o sociales, particularmente aquellos que son más frecuentes en la población. Esta dimensión se evalúa con respecto a:

G. Servicios disponibles: son aquellos con los que el centro cuenta para ser provistos cuando sea necesario. Secundarias

H. Servicios proporcionados: son servicios necesarios para la población, que el centro realmente provee.

I. Enfoque familiar: centrar la salud de los individuos en su contexto social más cercano.

J. Orientación comunitaria: dirigir la atención a resolver los problemas de salud de la comunidad, a la cual tiene como población diana.

K. Competencia cultural: adaptar y establecer relaciones que faciliten la atención de los problemas de salud en las personas de los diferentes grupos sociales de la población asignada.

Fuente: elaboración de los autores con base en la referencia 9.

a Siglas en inglés de "instrumentos para evaluar la atención primaria de salud".

b Las preguntas de la subdimensión "grado de afiliación" se ubican al inicio del cuestionario para identificar al proveedor de APS, y computa una suma, no un promedio, como el resto de dimensiones.

c De las 13 preguntas sobre "integración de la información", nueve computan el promedio de la dimensión.

riencia con su profesional o centro habitual de APS con respecto a cada atributo. El resto de las preguntas del cuestionario permiten identificar la fuente habitual de atención, la fuerza de dicha afiliación, y la caracterización de la persona que responde y la de su cobertura sanitaria.

\section{Análisis de los datos}

En primer lugar se examinaron los ítems y la validez factorial para considerar la remoción o inclusión de preguntas hasta decidir una versión definitiva del instrumento $\mathrm{y}$, entonces, realizar el análisis de ítems y de fiabilidad de las escalas de cada dimensión con la composición final del instrumento.

El análisis inicial de ítems incluyó las distribuciones de las respuestas de cada ítem, para observar si alguna opción acumulaba una proporción muy alta de respuestas en la categoría "no sabe"; la correlación de cada ítem con la puntuación total de su dimensión, que debería ser $>0,30$, y la correlación de cada ítem con otras dimensiones, que debería ser siempre $<0,20$, o bien, menor a la correlación con su propia dimensión.

La validez factorial se evaluó mediante la técnica del análisis factorial exploratorio. Se usó la técnica de extracción de componentes que produce factores capaces de reducir la información de todos los datos, esperando que cada factor se componga de los ítems propuestos para cada dimensión y explicando el concepto correspondiente a esa dimensión. Se forzó la extracción de siete a 12 factores para examinar cuál sería la solución más ajustada a los datos, teniendo en cuenta que el modelo teórico se puede componer de siete factores (dimensiones) a 10 factores, así como los conceptos de las subdimensiones. Como indicadores de validez del modelo se consideraron los siguientes criterios: factores que tuvieran tres o más ítems con una carga factorial suficiente $(>0,35)$ o una carga secundaria aceptable $(>0,20)$; correlación ítem-total moderada o alta $(>0,30)$; interpretación de la solución en consecuencia con el modelo teórico, aspecto que fue discutido por el equipo local con investigadores que elaboraron la versión homóloga para Brasil y la autora del modelo teórico y del instrumento (todos/as autores/as del presente trabajo).

A continuación, la conformación definitiva del instrumento fue sometida al análisis de fiabilidad de las escalas. En cada dimensión se examinaron los porcentajes de efecto suelo y techo (esperado $<15 \%$ ), medias, desviaciones estándar y porcentajes de ítems de cada dimensión que tienen una correlación ítem-total >0,30; también se evaluó la "razón de éxito de la escala", como el cociente entre el número de correlaciones en que los ítems de esta dimensión han sido superiores a las correlaciones de cada uno de estos ítem con las otras dimensiones y el total de correlaciones efectuadas para esta dimensión (dado por el número de ítems de esta dimen- 
sión por el número de dimensiones). Finalmente, se evaluó la consistencia interna del instrumento con el coeficiente $\alpha$ de Cronbach, que estima la proporción de varianza total de la escala que es atribuible a una fuente común, presumiblemente el verdadero rasgo latente en esa dimensión. Para su interpretación se adoptó el criterio $\alpha<0,50$ : insuficiente; 0,50 a 0,70 : moderado, y > 0,70: bueno. Los análisis se realizaron con la versión 18 del programa Statistical Package for the Social Science (SPSS).

El protocolo fue aprobado por el Comité Institucional de Ética de la Investigación en Salud Mental, Ministerio de Salud de la Provincia de Córdoba. Los aspectos éticos considerados fueron los de consentimiento informado, confidencialidad y protección de los datos.

\section{RESULTADOS}

\section{Descripción de la muestra}

La muestra quedó constituida por 296 casos, de los cuales $54 \%$ provenían del estudio en la obra social (cuadro 2). La proporción de respuesta en la muestra de madres y padres de escolares fue de $0,49(137 / 275$ que aceptaron participar) y, en la obra social, 0,64 (159/261 que aceptaron participar). Fue mayor la participación de mujeres $(73,6 \%)$ y de personas adultas jóvenes $(61,7 \%)$. El $45 \%$ de las madres y padres declaró que tenía solo cobertura pública y 55\%, de una obra social y/o de una empresa privada, mientras que entre los afiliados de la obra social, $25 \%$ declaró tener una cobertura adicional y siete personas declararon tener solo cobertura pública (un error de declaración admisible).

\section{Análisis de ítems y validez factorial}

El análisis factorial mostró que una solución de nueve factores fue la mejor al explicar un 57,3\% de la variabilidad de los datos y ajustarse adecuadamente al modelo teórico, aunque cuatro de los 25 ítems de la dimensión de globalidad (vacunaciones, control odontológico, tratamiento odontológico y prueba de Papanicolaou) formaron otro factor, $y$ los tres ítems de la subdimensión de sistemas de información no integraron un factor único sino que cargaron en otro. Teniendo en cuenta los análisis de ítems y los criterios establecidos para conformar la medida, se excluyeron los ítems G4 y D14 por presentar carga factorial $<0,35$ y correlación ítem-total $<0,30$ (cuadro 3).

En la siguiente fase, los análisis de ítems y factorial exploratorio se repitie-

CUADRO 2. Distribución de las personas incluidas en las dos submuestras y en la muestra total, para evaluar la atención primaria de salud (APS) con el cuestionario PCAT, ${ }^{a}$ por sexo, edad, estado de salud percibido y tipo de cobertura sanitaria, Córdoba, Argentina, 2010

\begin{tabular}{|c|c|c|c|c|c|c|}
\hline \multirow[b]{2}{*}{ Datos } & \multicolumn{2}{|c|}{$\begin{array}{c}\text { Madres y padres } \\
\text { institutos secundarios }\end{array}$} & \multicolumn{2}{|c|}{$\begin{array}{l}\text { Afiliados } \\
\text { obra social }\end{array}$} & \multicolumn{2}{|c|}{ Total } \\
\hline & No. & $\%$ & No. & $\%$ & No. & $\%$ \\
\hline \multicolumn{7}{|l|}{$\operatorname{Sexo}^{b}$} \\
\hline Mujeres & 124 & 90,5 & 94 & 59,1 & 218 & 73,6 \\
\hline Hombres & 13 & 9,5 & 65 & 40,9 & 78 & 26,4 \\
\hline \multicolumn{7}{|l|}{ Edad (años) ${ }^{b}$} \\
\hline $18-45$ & 102 & 74,5 & 77 & 50,3 & 179 & 61,7 \\
\hline $46-65$ & 34 & 24,8 & 49 & 32,0 & 83 & 28,6 \\
\hline$\geq 66$ & 1 & 0,7 & 27 & 17,6 & 28 & 9,7 \\
\hline \multicolumn{7}{|l|}{ Estado de salud } \\
\hline Bueno & 113 & 82,5 & 139 & 89,1 & 252 & 86,0 \\
\hline Malo & 24 & 17,5 & 17 & 10,9 & 41 & 14,0 \\
\hline \multicolumn{7}{|c|}{ Cobertura sanitariab } \\
\hline Solo pública & 62 & 45,3 & 7 & 4,4 & 69 & 23,3 \\
\hline Obra social & 41 & 29,9 & 112 & 70,4 & 153 & 51,7 \\
\hline Privada & 14 & 10,2 & 0 & 0,0 & 14 & 4,7 \\
\hline Doble $^{c}$ & 20 & 14,6 & 40 & 25,2 & 60 & 20,3 \\
\hline Total & 137 & 100,0 & 159 & 100,0 & 296 & 100,0 \\
\hline
\end{tabular}

a Siglas en inglés de "instrumentos para evaluar la atención primaria de salud".

b Valor $P<0,05$ para la prueba de hipótesis de diferencia de distribución entre las dos submuestras.

c Cobertura por parte de dos obras sociales o una obra social más una privada.

ron, incluyendo las 13 preguntas que el grupo de trabajo local propuso añadir durante el proceso de adaptación. Once de ellos cumplieron los criterios de carga factorial y correlación establecidos, por lo que seis fueron seleccionados tras considerar que aportarían información original sobre rasgos importantes en las dimensiones de servicios disponibles (entrega de métodos anticonceptivos, tratamiento de problemas de salud mental, tratamiento de adicciones y asesoramiento ante crisis vitales o cambios evolutivos) y orientación comunitaria (reuniones con instituciones del barrio y actividades de participación comunitaria), sin aumentar excesivamente la extensión del cuestionario (cuadro 3). Al realizar el análisis factorial exploratorio con los 96 ítems definitivos, la solución de nueve factores explicó $57,4 \%$ de la variabilidad de los datos. En esa extracción se observó que todos los ítems cargaron principalmente, o con una carga secundaria aceptable, en un factor común con los demás ítems que componían a priori la misma dimensión (ver anexo).

El análisis de fiabilidad de las escalas mostró que la mayoría de los ítems y también las dimensiones lograron un buen escalonamiento de las respuestas (cuadro 4). Las dimensiones de competencia cultural, primer contacto-utilización y coordinación-sistemas de información presentaron efecto techo, es decir, más de $15 \%$ de las personas puntuaron en el nivel máximo de la escala. El análisis de correlaciones ítem-total mostró que la convergencia no fue óptima en las dimensiones primer contacto-utilización y coordinación-sistemas de información, pero fue suficiente para el resto de las dimensiones. En cuanto a la correlación entre los ítems de una dimensión y los ítems de otras dimensiones, solo hubo dos casos, en las dimensiones continuidad y coordinación, que tuvieron una correlación algo mayor con otras dimensiones diferentes que aquella a la que aportan información según el modelo teórico, llevando la razón de éxito de la escala a $98 \%$ en ambos casos. La consistencia interna de las dimensiones fue buena (coeficiente $\alpha$ de Cronbach $>0,70)$ en la mayoría de ellas y no superó este estándar, pero fue suficiente en tres de ellas: primer contacto-utilización $(\alpha=0,59)$, coordinación-sistemas de información $(\alpha=0,50)$ y enfoque familiar $(\alpha=0,66)$. 
CUADRO 3. Resultados generales del análisis factorial y de correlación de ítems con las escalas de cada componente del cuestionario PCAT, ${ }^{a}$ que evalúa las dimensiones y subdimensiones de la atención primaria de salud (APS)

\begin{tabular}{|c|c|c|c|c|}
\hline \multirow[b]{2}{*}{ Factor $^{b}$} & \multirow[b]{2}{*}{ Dimensión (subdimensión) } & \multicolumn{2}{|c|}{ No. de ítems ${ }^{c}$} & \multirow[b]{2}{*}{ Observaciones } \\
\hline & & Piloto & Final & \\
\hline 1 & $\begin{array}{l}\text { G. Integralidad (servicios } \\
\text { disponibles) }\end{array}$ & 35 & 28 & $\begin{array}{l}\text { G2, G4, G5, G13 cargan en factor } 7 \text {. Por } \\
\text { correlación ítem-total se eliminó G4 }(0,24) \text { y } \\
\text { se mantuvo G2 }(0,39) \text {, G5 }(0,32) \text { y G13 }(0,50) \text {. } \\
\text { Se integró } 4 \text { de los } 11 \text { ítems nuevos (entrega } \\
\text { de métodos anticonceptivos, tratamiento de } \\
\text { problemas de salud mental, tratamiento de } \\
\text { adicciones, asesoramiento ante crisis vitales) } \\
\text { que cumplen criterios (ver anexo 1). }\end{array}$ \\
\hline \multirow[t]{2}{*}{2} & $\begin{array}{l}\text { D. Continuidad (relación } \\
\text { interpersonal) }\end{array}$ & 15 & 14 & $\begin{array}{l}\text { D14 (¿Podría usted cambiar de CAPS?) } \\
\text { presentó carga factorial }-0,184 \text { y correlación }\end{array}$ \\
\hline & I. Enfoque familiar & 3 & 3 & $\begin{array}{l}\text { ítem-total }-0,25 \text {. Se eliminó y pasó a la sección } \\
\text { de caracterización de la fuente de APS. }\end{array}$ \\
\hline 3 & $\begin{array}{l}\text { H. Integralidad (servicios } \\
\text { recibidos) }\end{array}$ & 12 & 12 & $\ldots e^{e}$ \\
\hline 4 & J. Orientación comunitaria & 9 & 8 & $\begin{array}{l}\text { Integró adecuadamente } 2 \text { de los } 3 \text { ítems nuevos } \\
\text { (reuniones con instituciones; actividades de } \\
\text { participación comunitaria) que cumplían criterios } \\
\text { (ver anexo 1). }\end{array}$ \\
\hline 5 & C. Primer contacto (acceso) ${ }^{f}$ & 7 & 7 & $r^{-1}$ \\
\hline 6 & $\begin{array}{l}\text { E. Coordinación (integración de } \\
\text { la información) }\end{array}$ & 9 & 9 & $\begin{array}{l}\text { E9 (¿Alguien que trabaja en su CAPS }{ }^{d} \text { lo } \\
\text { ayudó a conseguir el turno para esa consulta?) } \\
\text { presentó correlación ítem-total al límite }(0,29) \text { y } \\
\text { se mantiene. }\end{array}$ \\
\hline 7 & Ítems G2, G4, G5, G13 & & & Ver factor 1 \\
\hline 8 & B. Primer contacto (utilización) & 3 & 3 & $\ldots$ \\
\hline \multirow[t]{3}{*}{9} & C. Primer contacto (acceso) ${ }^{f}$ & 5 & 5 & $\ldots$ \\
\hline & K. Competencia cultural & 3 & 3 & \\
\hline & $\begin{array}{l}\text { F. Coordinación (sistemas de } \\
\text { información) }\end{array}$ & 3 & 3 & $\begin{array}{l}\text { Los } 3 \text { ítems cargaron en otras dimensiones; F1 y } \\
\text { F2 presentaron baja correlación ítem-total }(0,19 \\
\text { y } 0,27) \text {. }\end{array}$ \\
\hline
\end{tabular}

a Siglas en inglés de "instrumentos para evaluar la atención primaria de salud";

b Número de orden del factor extraído.

c Cantidad de ítems incluidos en la versión utilizada (estudios piloto) y propuestos para la versión final del cuestionario.

dCAPS: Centro o profesional de atención primaria de salud.

${ }^{\mathrm{e}}$ (... .): no registra observaciones.

${ }^{\dagger}$ Las preguntas de la subdimensión de acceso / primer contacto, mostraron cargas suficientes separadas en dos factores.

\section{DISCUSIÓN}

El presente trabajo examina las propiedades métricas de un instrumento para evaluar la APS desde la perspectiva de la población y sobre la base de un modelo conceptual de amplia aceptación y utilización a nivel internacional.

El conjunto PCAT se ha convertido en una herramienta altamente recomendada para la evaluación de sistemas y servicios de salud, pero su implementación requiere el conocimiento previo de su equivalencia con la versión original y la comprobación a través de la descripción de sus características métricas. Actualmente, una de las dificultades del uso internacional de estos instrumentos es la magnitud de los cambios que suelen sufrir cuando son adaptados a diferentes contextos sin atender a recomendaciones sobre este tipo de procesos.

En el caso de este estudio, la línea de investigación se inició con un cuidadoso proceso de adaptación cultural del conjunto PCAT (31), que incluyó técnicas de traducción directa e inversa (traducción y retro-traducción), varios momentos de consenso en el que participaron expertos locales, la autora del modelo teórico sobre el que se basa la medición y los autores de este trabajo, así como instancias de evaluación de la comprensión de preguntas por parte de la población mediante métodos cualitativos. De esta manera, se intentó conseguir cuestionarios adecuados

CUADRO 4. Propiedades métricas de las escalas de la versión argentina del PCAT-AEa

\begin{tabular}{|c|c|c|c|c|c|c|c|c|c|c|c|c|}
\hline \multirow[b]{2}{*}{ Dimensión/subdimensión } & \multirow[b]{2}{*}{ No. ${ }^{d}$} & \multirow[b]{2}{*}{$\begin{array}{c}\text { No. } \\
\text { ítems }\end{array}$} & \multicolumn{6}{|c|}{$\begin{array}{l}\text { Puntuación } \\
\text { dimensión }\end{array}$} & \multicolumn{2}{|c|}{$\begin{array}{l}\text { Correlación } \\
\text { ítem-totalc }^{c}\end{array}$} & \multirow{2}{*}{$\begin{array}{c}\text { Razón de éxito } \\
\text { de escala } \\
(\%)\end{array}$} & \multirow[b]{2}{*}{$\begin{array}{c}\alpha \text { de } \\
\text { Cronbach }\end{array}$} \\
\hline & & & Mín & Máx & Media & $\mathrm{DE}^{f}$ & $\begin{array}{l}\text { Suelo } \\
(\%)\end{array}$ & $\begin{array}{c}\text { Techo } \\
(\%)\end{array}$ & $\begin{array}{c}>0,30 \\
(\%)\end{array}$ & Rango & & \\
\hline B - Primer contacto/Utilización & 283 & 3 & 1,0 & 4,0 & 3,1 & 0,7 & 1,8 & 17,1 & 67 & $0,16-0,46$ & 100 & 0,51 \\
\hline C - Primer contacto/Acceso & 242 & 12 & 1,1 & 4,0 & 2,8 & 0,6 & 0,0 & 0,4 & 92 & $0,25-0,62$ & 100 & 0,79 \\
\hline D - Continuidad & 262 & 14 & 1,2 & 4,0 & 3,0 & 0,6 & 0,4 & 1,2 & 100 & $0,43-0,74$ & 98 & 0,90 \\
\hline E - Coordinación/Integración de información & 213 & 9 & 1,0 & 4,0 & 2,9 & 0,8 & 0,9 & 4,7 & 78 & $0,13-0,67$ & 98 & 0,80 \\
\hline F - Coordinación/Sistemas de información & 284 & 3 & 1,0 & 4,0 & 3,1 & 0,7 & 0,7 & 18,0 & 67 & $0,19-0,42$ & 100 & 0,44 \\
\hline G - Integralidad/Servicios disponibles & 237 & 28 & 1,1 & 4,0 & 2,9 & 0,6 & 0,0 & 2,5 & 96 & $0,28-0,78$ & 100 & 0,95 \\
\hline H - Integralidad/Servicios recibidos & 258 & 12 & 1,0 & 4,0 & 2,6 & 0,8 & 6,2 & 5,0 & 100 & $0,45-0,74$ & 100 & 0,89 \\
\hline I- Enfoque familiar & 280 & 3 & 1,0 & 4,0 & 2,5 & 0,9 & 12,5 & 9,6 & 100 & $0,40-0,51$ & 100 & 0,66 \\
\hline $\mathrm{J}$ - Orientación comunitaria & 268 & 8 & 1,0 & 4,0 & 2,2 & 0,6 & 5,6 & 1,1 & 100 & $0,33-0,69$ & 100 & 0,83 \\
\hline K - Competencia cultural & 286 & 3 & 1,0 & 4,0 & 3,2 & 0,7 & 1,0 & 28,7 & 100 & $0,46-0,58$ & 100 & 0,70 \\
\hline
\end{tabular}

a PCAT-AE: Siglas en inglés de "instrumentos para evaluar la atención primaria de salud, versión para usuarios adultos".

b Valores mínimo, máximo, media, desviación estándar y porcentaje de casos que puntúa en los valores mínimo (suelo) y máximo (techo) de la escala.

c Correlación ítem-total: porcentaje de ítems con correlación $>0,30$ y rango de valores obtenidos por los ítems de las escalas.

${ }^{d}$ Cantidad de respuestas incluidas en el análisis.

e Cantidad de preguntas que componen la dimensión.

f DE: Desviación estándar. 
a la población y al sistema sanitario local, a la vez que equivalentes a las versiones originales y a las de otros países que realizaron procesos similares. El análisis de fiabilidad y validez que se proporciona aquí sirvió para llegar a una versión definitiva, alterando mínimamente el cuestionario luego de discutir ítem por ítem sobre la base de criterios conceptuales y técnicos. Si bien se puede considerar que el instrumento obtenido consigue el objetivo de equivalencia, validez y fiabilidad adecuadas, a continuación se discuten varias circunstancias que deben tenerse en cuenta como limitaciones del proceso y del instrumento logrado.

Esta versión de los PCAT adaptada para Argentina mantiene la composición de la edición extensa de los instrumentos, con un reemplazo de pocos ítems que producen cambios de diversa importancia en el contenido. Un ítem de la dimensión de continuidad fue eliminado con el acuerdo de la autora, tras comprobar su falta de homogeneidad con otros de esa dimensión, además de que se trata de un rasgo que no se releva en los cuestionarios PCAT para profesionales y centros. En cuanto a los seis ítems agregados, indagan aspectos concretos de la disponibilidad de servicios de salud reproductiva y mental en la dimensión de integralidad, y profundizan en detalle acerca de la orientación comunitaria. Estos nuevos ítems cubren prioridades destacadas en América Latina $(6,32)$; algunos de ellos también han sido incluidos en otros procesos de adaptación de los PCAT (26) y representan una contribución a la discusión actual acerca de la amplitud del concepto de integralidad de los servicios. Estos ítems deberían ser desestimados en estudios internacionales, por ser específicos para la edición argentina y la diferencian de otras versiones de los cuestionarios PCAT. El grupo de trabajo local había propuesto adicionar más ítems (31) que resultaron métricamente correctos, pero fueron descartados para no aumentar la carga de administración del cuestionario por la adición de nuevas preguntas.

La subdimensión de sistemas de información en la función de coordinación no mostró una validez factorial adecuada. Estos resultados, que coinciden con las pruebas al cuestionario realizadas en Brasil, podrían deberse a la infrecuente disponibilidad de recursos con que se cuenta en el ámbito latinoamericano en general, y en las muestras utilizadas en particular. Dada la importancia de esta dimensión en el modelo conceptual, se considera necesario mantener tales preguntas en el cuestionario, a la vez que es recomendable continuar probando su validez en estudios futuros.

Por otra parte, varias preguntas de la versión argentina para usuarios adultos generaron muchas respuestas en la opción "no sabe" en ítems relativos a atributos que posiblemente los usuarios no conozcan, o bien que son infrecuentes en algunos sectores del sistema sanitario de este país, tales como recibir atención médica telefónica o que alguien del centro le ayude a conseguir un turno con un servicio especializado. A pesar del cuidadoso proceso de adaptación realizado, es probable que algunas preguntas aún planteen dificultades de comprensión, aunque consigan propiedades métricas adecuadas. Estas y otras debilidades son admisibles por tratarse de un instrumento que intenta recoger numerosos y diversos rasgos de la experiencia de las personas con los servicios de salud en cualquier sector y a la vez mantener la equivalencia con cuestionarios homólogos de otros países.

La representatividad de la muestra del presente estudio es limitada respecto de la población general, pero tal rasgo no debería representar una limitación para analizar la validez y la fiabilidad de una herramienta de evaluación como los PCAT. Se tomaron muestras de dos poblaciones que aportaron variabilidad en cuanto a la proporción en diferentes opciones de cobertura de salud, tal como sucede en la población argentina y de manera similar a lo realizado en la evaluación de la versión original (10). Ninguna de las muestras se restringió a usuarios de servicios sanitarios para incluir a personas con diversos niveles de acceso y volumen de utilización, aspectos que se asocian a las experiencias que luego se declaran respecto de la atención primaria de salud (33). Así, este trabajo proporciona información que podría ser comprobada en futuros estudios sobre muestras representativas, pudiéndose suponer que las diferencias en cuanto a las pruebas realizadas no serían significativas.

\section{Conclusiones y recomendaciones}

La estructura final propuesta para la versión argentina del cuestionario PCAT dirigido a personas adultas logró validez y fiabilidad adecuadas, teniendo en cuenta su composición factorial, las propiedades de los ítems y su consistencia interna. Esta comprobación de las propiedades métricas del instrumento no solo ofrece garantías de calidad de la medición, sino que también muestra un procedimiento de adaptación y evaluación de cuestionarios que podría ser tenido en cuenta por futuros grupos de trabajo en otros países. Esta versión (AR-PCAT-AE) no puede aplicarse en otros países sin previa adaptación. El presente informe puede servir como referencia para realizar procesos similares en otros contextos, tanto por los procedimientos como por los criterios empleados para tomar decisiones, ya que reunió la experiencia de la autora de la versión original y de equipos que realizaron trabajos similares en Brasil y España.

La utilidad del cuestionario PCAT se fundamenta en el interés por obtener información original para la valoración de los servicios de salud y la posterior reflexión acerca de su desempeño, así como en que hace posible discutir aspectos conceptuales y metodológicos de la evaluación de servicios. Existen pocas herramientas que permitan obtener indicadores de las funciones de la APS desde la perspectiva de la población, aportando información sobre conceptos que gozan de amplio y aún creciente consenso a nivel internacional.

Idealmente, la implementación de este cuestionario debería complementarse con otras perspectivas y métodos de investigación. Algunas posibilidades enriquecedoras serían la administración simultánea a profesionales de salud para obtener también sus puntos de vista; el examen de resultados en salud para observar si se correlacionan con el nivel de cumplimiento de las funciones de la APS, y estudios cualitativos dirigidos a comprender procesos y mecanismos que pudieran explicar diferentes resultados en el logro de las funciones de la APS.

Agradecimientos. Los autores quieren agradecer a las personas que participaron en la realización del presente estudio, así como a las autoridades de la Obra Social Universitaria DASPU y a las de los institutos de enseñanza media que autorizaron, respectivamente, el contacto con afiliados, y madres y padres de los alumnos. También desean expresar su gratitud a Cristina Somazzi (Programa de Estadísticas Universitarias, Secretaría 
Académica, Universidad Nacional de Córdoba) por el desarrollo de la versión digital del cuestionario y su apoyo en la recolección de los datos por este medio; a Verónica Mamondi y Romina Vitale, por su asistencia en el trabajo de campo en los institutos de enseñanza media; a Alicia Villalba Constanza, Falá Giroldi, Carolina Bernardó y al personal de las áreas de Informática, Afiliaciones y Co- municaciones de la Obra Social Universitaria por su asistencia en el trabajo de campo en esta entidad.

Financiamiento. El presente trabajo fue financiado por una beca Ramón Carrillo-Arturo Oñativia 2009/2010 (Comisión Nacional Salud Investiga, Ministerio de Salud), subsidios de la Secretaría de Ciencia y Tecnología (SECYT)

\section{REFERENCIAS}

1. World Health Organization. World Health Report 2008: Primary health care: now more than ever. Geneva: World Health Organization; 2008.

2. Pan American Health Organization. Renewing Primary Health Care in the Americas: A Position Paper of the Pan American Health Organization/WHO. Washington, DC: $\mathrm{PAHO} / \mathrm{WHO} ; 2005$.

3. Macinko J, Montenegro H, Nebot Adell C, Etienne C, Grupo de Trabajo de Atención Primaria de Salud de la Organización Panamericana de la Salud. La renovación de la atención primaria de salud en las Américas. Rev Panam Salud Publica. 2007;21(2-3): 73-84.

4. Organización Panamericana de la Salud. Declaración regional sobre las nuevas orientaciones de la atención primaria de salud. Montevideo, $46^{\circ}$ Consejo Directivo, $57^{a}$ sesión del Comité Regional. Washington, D.C.: OPS; 2005.

5. Organización Panamericana de la Salud/ Organización Mundial de la Salud (OPS/ OMS). La renovación de la atención primaria de salud en las Américas. Washington DC: Organización Panamericana de la Salud/ Organización Mundial de la Salud; 2007.

6. Haggerty JL, Yavich N, Báscolo EP. Grupo de consenso sobre un marco de evaluación de la atención primaria en América Latina. Rev Panam Salud Publica. 2009;26(5):377-84.

7. Macinko J, Starfield B, Erinosho T. The impact of primary healthcare on population health in low- and middle-income countries. J Ambul Care Manage. 2009;32(2):150-71.

8. Donaldson MS, Yordy KD, Vanselow NA. Institute of Medicine: Defining Primary Care: an interim report. Washington, DC: National Academy Press; 1994.

9. Starfield B. Primary Care: Balancing Health Needs, Services and Technology. New York: Oxford University Press; 1998.

10. Shi L, Starfield B, Xu J. Validating the Adult Primary Care Assessment Tool. J Fam Pract. 2001;50(2):161.

11. Cassady CE, Starfield B, Hurtado MP, Berk RA, Nanda JP, Friedenberg LA. Measuring Consumer Experiences with Primary Care. Pediatrics. 2000;105:998-1003.

12. Malouin RA, Starfield B, Sepulveda MJ. Evaluating the Tools Used to Assess the Medical Home. Manag Care. 2009;18(6):44-8.

13. Starfield B, Shi L. PCAT Manual. Baltimore: Johns Hopkins University; 2009.
14. Haggerty J, Martin CM. Evaluating Primary Health Care in Canada and The Right Questions to Ask! The National Evaluation Strategy for Primary Health Care. Ottawa, Canadá: Health Canada; 2005.

15. Haggerty JL, Pineault R, Beaulieu MD, Brunelle Y, Gauthier J, Goulet F, et al. Patients' experiences of primary care in Quebec before major reforms. Can Fam Physician. 2007;53:1056-57.

16. Macinko J, Almeida C, Klingelhoefer de Sa P. A Rapid Assessment Methodology for the Evaluation of Primary Care Organization and Performance in Brazil. Health Policy Plan. 2007;22(3):167-77.

17. Harzheim E, Starfield B, Rajmil L, ÁlvarezDardet C, Stein AT. Consistência interna e confiabilidade da versão em português do instrumento de avaliação da atenção primária (PCATool-Brasil) para serviços de saúde infantil. Cad Saude Publica. 2006;22:1649-59.

18. Harzheim E, Rodrigues Gonçalves M, Oliveira M, Gomes da Trindade T, Rodrigues Agostinho M, Hauser L. Manual do Instrumento de Avaliação da Atenção Primária à Saúde. Brasilia DF: Ministerio da Saúde; 2009.

19. Pasarín MI, Berra S, Rajmil L, Solans M, Borrell C, Starfield B. Un instrumento para la evaluación de la atención primaria de salud desde la perspectiva de la población. Aten Primaria. 2007;39(8):395-401.

20. Pasarín MI, Rocha KB, Rodríguez-Sanz M, Berra S, Borrell C. Un paso más en el estudio de las desigualdades sociales en la provisión de atención primaria desde la perspectiva de la población. Med Clin (Barc). 2011;137(supl 2):49-54.

21. Pasarín MI, Berra S, González A, Segura A, Tebé C, García-Altés A, et al. Evaluation of primary care: The "Primary Care Assessment Tools-Facility version" for the Spanish health system. Gac Sanit. 2012 Aug 23. [Epub ahead of print].

22. Wong SY, Kung K, Griffiths SM, Carthy T, Wong MC, Lo SV, et al. Comparison Of Primary Care Experiences Among Adults in General Outpatient Clinics and Private General Practice Clinics in Hong Kong. BMC Public Health. 2010;10:397.

23. Jatrana S, Crampton P, Richardson K. Continuity of care with general practitioners in New Zealand: results from SoFIE-Primary Care. N Z Med J. 2011;124(1329):16-25.

24. Tsai J, Shi L, Yu WL, Hung LM, Lebrun LA. Physician specialty and the quality of medical de la Universidad Nacional de Córdoba y el Consejo Nacional de Investigaciones Científicas y Técnicas (CONICET) de Argentina, y del Programa Telessaúde, Secretaria de Gestão da Educação e do Trabalho na Saúde, Ministerio de Salud, Brasil.

Conflicto de intereses. Ninguno declarado pos los autores. care experiences in the context of the Taiwan national health insurance system. J Am Board Fam Med. 2010;23(3):402-12.

25. Tsai J, Shi L, Yu WL, Lebrun LA. Usual source of care and the quality of medical care experiences: a cross-sectional survey of patients from a Taiwanese community. Med Care. 2010;48(7):628-34.

26. Pizzanelli M, Ponzo J, Buglioli M, Toledo A, Casinelli M, Gómez A. Validación de Primary Care Assessment Tool (PCAT) en Uruguay. Rev Med Urug. 2011;27(3):187-9.

27. Rocha KB, Rodríguez-Sanz M, Pasarín MI, Berra S, Gotsens-Miquel M, Borrell C. Assessment of primary health care in health surveys: a population perspective. Eur J Public Health. 2011. 22(1):14-9. Apr 5. [Epub ahead of print].

28. Berra S, Rocha KB, Rodríguez-Sanz M, Pasarín MI, Rajmil L, Borrell C, et al. Properties of a short questionnaire for assessing Primary Care experiences for children in a population survey. BMC Public Health. 2011;11(1):285.

29. Stolkiner A. Neoliberalismo y servicios de salud en la Argentina: estudio de caso. Quadern CAPS. 2001;30:8-16.

30. Argentina, Instituto Nacional de Estadísticas y Censos. Gacetilla de prensa del 29 de diciembre de 2011. Disponible en: http:// www.censo2010.indec.gov.ar/archivos/ novedades/gacetilla_29_12_11.pdf. Acceso el 23 de noviembre de 2012.

31. Berra S, Audisio Y, Mántaras J, Nicora V, Mamondi V, Starfield B. Adaptación del conjunto de instrumentos para la evaluación de la atención primaria de la salud (PCAT) al contexto argentino. Rev Arg Salud Publica. 2011;2(8):6-14.

32. Organización Panamericana de la Salud. Seminario-taller Internacional sobre Atención Primaria de Salud: a 25 años de Alma Ata. 1. ${ }^{a}$ ed. Buenos Aires: Organización Panamericana de la Salud; 2003.

33. Berra S, Pasarín MI, Rajmil L, RodríguezSanz M, Starfield B, Borrell C. Experiences with primary care for children in the Catalan population. 19th WONCA World Conference. Cancún, México, May 2010.

Manuscrito recibido el 10 de mayo de 2012. Aceptado para publicación el 12 de octubre de 2013. 
ANEXO 1. Ítems de la versión argentina del PCAT-AE, ${ }^{a}$ ordenados por factores extraídos mediante análisis de principales componentes: carga factorial y correlación ítem-total

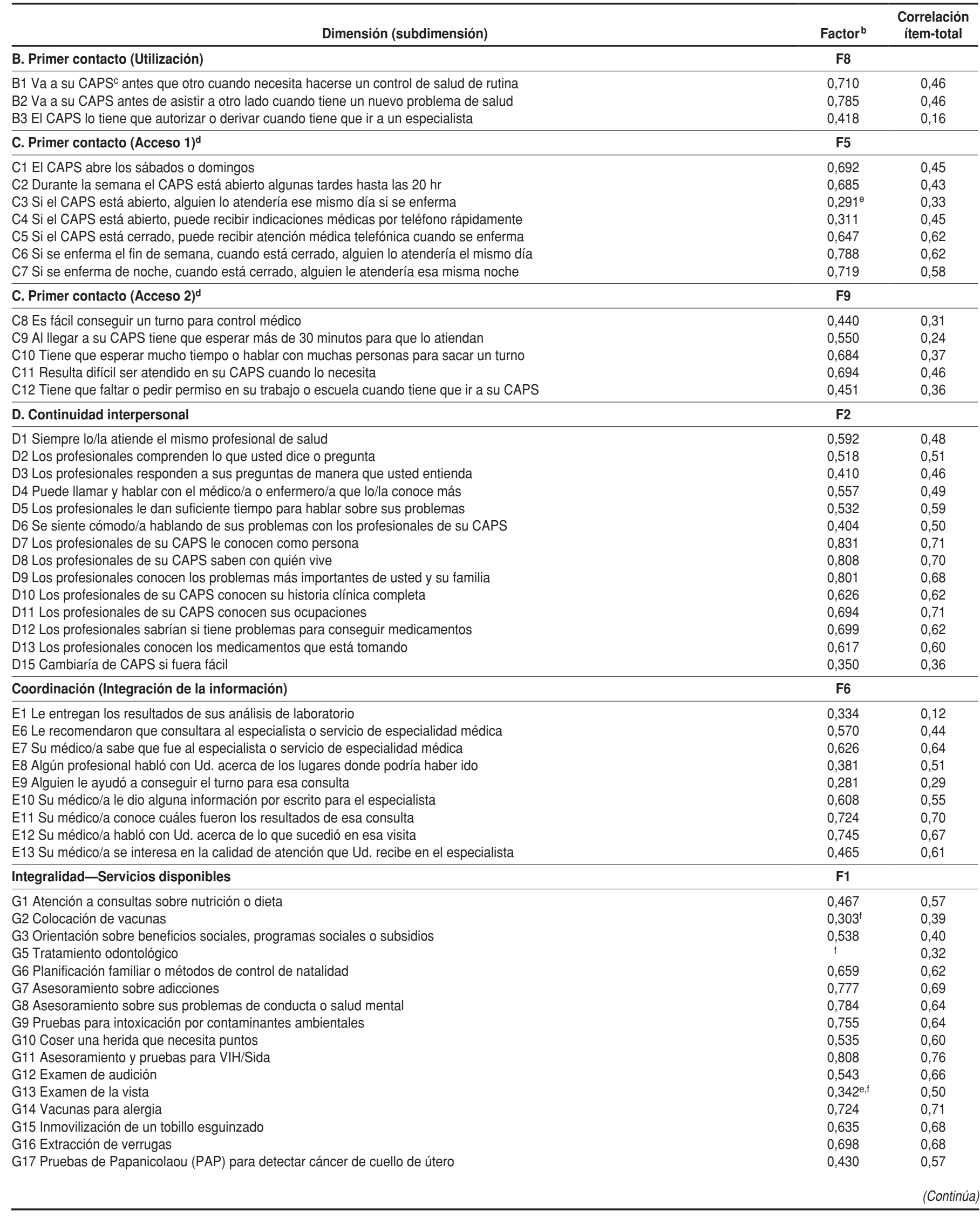


ANEXO 1. Continuación

\begin{tabular}{|c|c|c|}
\hline Dimensión (subdimensión) & Factor $^{b}$ & $\begin{array}{l}\text { Correlación } \\
\text { ítem-total }\end{array}$ \\
\hline G18 Exámenes de recto para detectar cáncer de intestino & 0,674 & 0,68 \\
\hline G19 Asesoramiento sobre el consumo de tabaco & 0,690 & 0,57 \\
\hline G20 Control del embarazo & 0,589 & 0,63 \\
\hline G21 Consultar por uñas encarnadas & 0,667 & 0,73 \\
\hline G22 Asesoramiento sobre discapacidades & 0,676 & 0,59 \\
\hline G23 Asesoramiento sobre envejecimiento & 0,769 & 0,68 \\
\hline G24 Asesoramiento acerca de cuidados de enfermería en el hogar & 0,718 & 0,59 \\
\hline G25 Control de niño sano y programas materno-infantiles & 0,538 & 0,63 \\
\hline G279 Entrega de métodos anticonceptivos & 0,631 & 0,50 \\
\hline G309 Tratamiento de problemas de salud mental & 0,860 & 0,73 \\
\hline G32g Tratamiento de adicciones & 0,783 & 0,53 \\
\hline G339 Asesoramiento ante crisis vitales o cambios evolutivos & 0,642 & 0,51 \\
\hline Integralidad (Servicios recibidos) & F3 & \\
\hline H1 Hábitos saludables, como alimentación y descanso & 0,402 & 0,60 \\
\hline H2 Seguridad del hogar & 0,829 & 0,67 \\
\hline H3 Modos de prevenir accidentes de tránsito y en la vía pública & 0,855 & 0,64 \\
\hline H4 Manejo de conflictos con otras personas & 0,583 & 0,62 \\
\hline H5 Ejercicio físico apropiado para usted & 0,307 & 0,46 \\
\hline H6 Control de niveles de colesterol en la sangre & 0,412 & 0,57 \\
\hline H7 Control y asesoramiento sobre los medicamentos que consume & 0,396 & 0,59 \\
\hline H8 Posible exposición a sustancias tóxicas en su casa, trabajo o barrio & 0,684 & 0,70 \\
\hline H10 Prevención de quemaduras & 0,793 & 0,69 \\
\hline H11 Prevención de caídas & 0,822 & 0,74 \\
\hline H12 Para mujeres: prevención de osteoporosis o huesos frágiles & $\mathrm{h}$ & 0,55 \\
\hline H13 Para mujeres: cuidados de molestias de la menstruación o la menopausia & $\mathrm{h}$ & 0,45 \\
\hline Enfoque familiar & F2 & \\
\hline $\begin{array}{l}\text { I1 Los profesionales preguntan opiniones e intenciones para planificar la atención y } \\
\text { tratamiento de alguien de la familia }\end{array}$ & 0,384 & 0,49 \\
\hline I2 Los profesionales conocen los problemas que pueden existir en la familia & 0,476 & 0,51 \\
\hline I3 Los profesionales se reunirían con alguien de la familia para ayudar a su tratamiento & 0,358 & 0,40 \\
\hline Orientación comunitaria & F4 & \\
\hline J1 Alguien del CAPS realiza visitas a domicilio & i & 0,35 \\
\hline J2 Los profesionales conocen los problemas de salud más importantes del barrio & 0,564 & 0,50 \\
\hline J3 Los profesionales aceptan opiniones e ideas que podrían mejorar la atención de salud & 0,543 & 0,44 \\
\hline J7 Encuestas a los pacientes sobre las necesidades de la gente & 0,694 & 0,55 \\
\hline J8 Encuestas a los integrantes de la comunidad sobre sus problemas de salud & 0,712 & 0,66 \\
\hline J9 Se invita a alguien del barrio a participar de las reuniones del equipo de salud & 0,696 & 0,60 \\
\hline J259 Los profesionales realizan reuniones con instituciones de la comunidad & 0,702 & 0,67 \\
\hline J269 Los profesionales realizan actividades de participación comunitaria & 0,744 & 0,60 \\
\hline Competencia cultural & F9 & \\
\hline K1 Le recomendaría su CAPS a un amigo o pariente & 0,403 & 0,57 \\
\hline K2 Le recomendaría su CAPS a una persona que no sepa hablar bien castellano o que viene de otro país & 0,448 & 0,58 \\
\hline K3 Le recomendaría su CAPS a una persona que utiliza hierbas o medicamentos caseros, o asiste al curandero & 0,431 & 0,45 \\
\hline
\end{tabular}

a PCAT-AE: Siglas en inglés de "instrumentos para evaluar la atención primaria de salud, versión para usuarios adultos". El texto de los ítems mantiene el contenido pero no es exactamente igual a las preguntas del cuestionario.

b Factores extraídos. Carga factorial de los ítems en el factor. Los factores con carga < 0,35 no se presentan en el cuadro, salvo cargas secundarias consideradas.

c CAPS: Centro o profesional de atención primaria de salud.

d Las preguntas de la subdimensión acceso/primer contacto, cargaron por separado en dos factores.

e Carga secundaria.

f G2 $(0,396)$, G5 $(0,831)$ y G13 $(0,481)$ cargan en el factor 7 .

$\mathrm{g}$ Ítem añadido en la versión argentina.

h No se incluyó en el análisis factorial por ser respondido solo por mujeres.

i J1 carga en F5 $(0,334)$. 
ABSTRACT Objective. To determine the validity and reliability of the version of the Primary Care Assessment Tool for adult users, adapted for Argentina (known as AR-PCAT-AE).

Validity and reliability of

Methods. Two cross-sectional pilot studies were conducted among people over age 17 , largely through self-administered questionnaires. Items that were equivalent to the original version of the PCAT and 13 others proposed for the instruments were analyzed. The factorial validity and reliability of the scores for each dimension were studied. The validity indicators were: factors that had three or more items with a factorial load of $>0.35$, item-total correlation $>0.30$, and interpretation of the solution according to the theoretical model.

Results. The factorial analysis yielded nine factors that explained $57.4 \%$ of the variability. Considering the pre-established criteria for validity, two items were excluded from the dimensions of continuity and comprehensiveness and six were added to comprehensiveness and cultural competency. In the dimensions, the percentage of questions with an item-total correlation of greater than 0.30 ranged from $67 \%$ to $100 \%$ and the Chronbach's (alpha) coefficient of internal consistency ranged from 0.44 to 0.90 .

Conclusions. The validity and reliability of the AR-PCAT-AE questionnaire are adequate, maintaining high equivalence with the original version. The process presented could be adopted in other contexts to advance the evaluation of primary health care functions with quality tools.

Key words Primary health care; health surveys; health services; ambulatory care; Argentina. 